\title{
LECTOTIPIFICACIÓN DE CEPHALANTHUS PERUVIANUS Y C. BREVIFLORUS (RUBIACEAE)
}

\author{
M. Florencia Romero', Roberto M. Salas ${ }^{1,2}$ \& Ana M. Gonzalez ${ }^{1,3}$
}

\author{
${ }^{1}$ Instituto de Botánica del Nordeste (UNNE-CONICET), Sargento Cabral 2131, C.P. 3400, Corrientes, C.C. 209, \\ Argentina; mariafloromero@gmail.com (autor corresponsal). \\ ${ }^{2}$ Facultad de Ciencias Exactas y Naturales y Agrimensura (FaCENA-UNNE), Avenida Libertad 5470, C.P. 3400. \\ Corrientes, Argentina. \\ ${ }^{3}$ Facultad de Ciencias Agrarias (UNNE), Sargento Cabral 2131, C. P. 3400, Corrientes, Argentina.
}

\begin{abstract}
Romero, M. F.; R. M. Salas \& A. M. Gonzalez. 2019. Lectotypification of Cephalanthus peruvianus and C. breviflorus (Rubiaceae). Darwiniana, nueva serie 7(1): 65-67.

As part of the review of the genus Cephalanthus for the Americas, we have found that names $C$. peruvianus and C. breviflorus need a properly lectotypification. For this reason, materials collected by Richard Spruce and deposited in BR are here selected as lectotypes.
\end{abstract}

Keywords. Ixora; lectotype; Peruvian Amazon.

Resumen. Romero, M. F.; R. M. Salas \& A. M. Gonzalez. 2019. Lectotipificación de Cephalanthus peruvianus y C. breviflorus (Rubiaceae). Darwiniana, nueva serie 7(1): 65-67.

Como parte de la revisión del género Cephalanthus para las Américas, hemos encontrado que los nombres C. peruvianus y C. breviflorus necesitan una adecuada lectotipificación. Por esta razón, los materiales recolectados por Richard Spruce y depositados en BR son aquí seleccionados como lectotipos.

Palabras clave. Amazonia peruana; Ixora; lectotipo.

\section{INTRODUCCIÓN}

El género Cephalanthus L. pertenece a la tribu Naucleeae, subtribu Cephalanthinae, y actualmente cuenta con 6 especies, tres habitan en América, una en África y dos en Asia (Ridsdale, 1976; Razafimandimbison \& Bremer, 2002). Cephalanthus peruvianus Spruce ex K. Schum. y C. breviflorus Spruce ex K. Schum. fueron simultáneamente publicadas por Schumann (1889) en la Flora Brasiliensis. Ambas especies fueron diferenciadas de Cephalanthus gla- bratus (Spreng.) K. Schum, única especie del género conocida hasta entonces para Sudamérica, por tener bractéolas lineares, corola eglandulosa y estigma bilobado. En contraste, $C$. glabratus y las otras dos especies americanas del género, C. occidentalis L. y C. salicifolius Bonpl., se caracterizan por tener bractéolas espatuladas, corola con una "glándula" entre los lóbulos (coléter corolino) y estigma clavado, tales diferencias son reconocidas actualmente como sinapomorfías del género Cephalanthus (Razafimandimbison \& Bremer, 2002). 
Posteriormente, durante el estudio de la Flora Boliviana, Cephalanthus peruvianus fue transferido a Ixora L. por Standley (1931), quien realizó la combinación Ixora peruviana (Spruce ex K. Schum.) Standl.; po$\cos$ años después el mismo autor (Standley, 1936) colocó a C. breviflorus en sinonimia de I. peruviana.

Ixora es un género pantropical, considerado como el segundo más grande de la familia Rubiaceae, después de Psychotria L, por tener más de 300 especies (Mouly et al., 2009). El género puede ser reconocido principalmente por la presencia de pecíolo articulado, además, son arbolitos o arbustos inermes, con hojas generalmente opuestas, frecuentemente sin domacios, estípulas triangulares, frecuentemente aristadas, inflorescencias terminales, multifloras, bracteadas, flores homostilas, fragantes, con 2 carpelos, cada lóculo 1-óvulado, con placentación axilar y frutos drupáceos, con cada mitad 1-seminada (Taylor et al., 2004; Taylor, 2012).

Durante la realización de una nueva revisión actualizada del género Cephalanthus, observamos que los nombres Cephalanthus breviflorus y Cephalanthus peruvianus no fueron taxonómicamente tratados desde los estudios realizados por Standley. Los materiales originales de ambos taxones fueron colectados por Spruce y, desde su descripción original, no se han designado adecuadamente sus tipos nomenclaturales.

\section{MATERIALES Y MÉTODOS}

Se siguieron técnicas convencionales de taxonomía. Los tipos nomenclaturales fueron examinados en visitas a los herbarios BR, F, $\mathrm{K}, \mathrm{MG}, \mathrm{NY}, \mathrm{G}, \mathrm{MO}$ y USZ, también fueron consultadas imágenes de alta calidad de ejemplares de herbarios BM, E, GH, LD, P, o TCD disponibles en Jstor (https://plants.jstor.org) y ejemplares en herbarios INPA y RB disponibles a través del herbario virtual Reflora (http://reflora.jbrj.gov.br/reflora/herbarioVirtual). Acrónimos de los herbarios de acuerdo a Thiers (2019).

\section{RESULTADOS}

Ixora peruviana (Spruce ex K. Schum.) Standl., Publ. Field Mus. Nat. Hist., Bot. Ser. 7: 296. 1931. Cephalanthus peruvianus Spruce ex K. Schum., Fl. Bras 6(6): 129. 1888. Lectotipo: Perú: San Martín, prope Tarapoto, in Peruviae orientali, IV-1855/1856, R. Spruce 4910 (BR cb 0000005317547!, aquí designado; isolectotipos BM cb 000777517, E cb 00285084!, F cb 0040812F!, GH cb 00092514!, K cb 000424570!, K cb 000424571!, LD cb 1219883!, NY cb 00131035!, RB cb 00543504 !.

Cephalanthus breviflorus K. Schum., F1. Bras. 6(6): 129. 1888. Lectotipo: Perú, San Martín, prope Tarapoto, Peruviae Orientali, IV-1855/1856, R. Spruce 4175 (BR cb 0000005317219 !, aquí designado; isolectotipos BM cb 000777518, E cb 00285082!, $\mathrm{G}$ cb 00300168!, GH cb 00092513, K cb 000424573, NY cb 00131034!, P cb 00836756!, RB cb 00543503!, TCD cb 0005597 !).

Arbustos de hasta $3 \mathrm{~m}$ alt.; hojas pecioladas, oblongas a oblongo-elípticas, 12-18 cm long., glabras o glabrescentes, con estípulas bien desarrolladas, enteras, subtriangulares a elípticas, acuminadas y externamente estrigosas. Inflorescencias congestas, bracteadas, cortamente pedunculadas, con pedúnculos pilosos y flores conspicuamente sésiles. Corola blanca, muy fragante, 3-10 mm long., similar a jazmín. Hipanto densamente piloso, con lóbulos calicinos muy pequeños o casi truncados.

Distribución y hábitat. De acuerdo a información disponible en Tropicos.org, se conoce actualmente que la especie habita en bosques húmedos de tierras firme en Bolivia (Beni, Cochabamba, La Paz, Pando y Santa Cruz), Brasil (Acre) y Perú (Loreto, Madre de Dios, San Martín, Ucayali).

Comentarios. El tipo de inflorescencia congesta y globosa, probablemente llevó a que 
fuera descripta por Schumann (1889) dentro del género Cephalanthus.

En cuanto a la corola, el análisis del tipo de ambos nombres reveló que su tamaño varía desde 3 a 6 mm long. en el tipo de Cephalanthus breviflorus, mientras que de 7,5 a $10 \mathrm{~mm}$ long. en el tipo de C. peruvianus. Esta diferencia en la longitud de las corolas apoya la decisión original de Schumann (1889) de describirlas como especies diferentes. Sin embargo, Standley $(1931,1936)$ las considera una sola especie, lo cual es comprobable a través del análisis de especímenes cubriendo el rango de distribución, donde se observa un continuum en este carácter. Por otro lado, ambos especímenes (Spruce 4910 y Spruce 4175) coinciden, entre otros aspectos, en tener el hipanto densamente piloso, con lóbulos calicinos muy pequeños o casi truncados.

Se eligieron como lectotipos los ejemplares BR 0000005317547 y BR 0000005317219 porque reúnen los caracteres diagnósticos del protólogo y por ser los mejores conservados, con presencia de flores, entre los duplicados encontrados. Además, ambos ejemplares pertenecen a la colección Martius depositada en $\mathrm{BR}$, con lo cual podría presumirse que fueron oportunamente estudiados por Schumann durante la preparación de las Rubiáceas para la Flora Brasiliensis.

El nombre Faramea congesta Huber [TIPO: Perú, Ucayali: Cerro de Canchahyaya, Río Ucayali, 02-XI-1898, J. Huber 1417 (holotipo MG cb 001417!, isotipo RB cb 00340513!) aparece en Tropicos.org como sinónimo de Ixora peruviana; sin embargo, el análisis del tipo mencionado revela que corresponde a un sinónimo de Alibertia bertierifolia K. Schum.

\section{Material representativo examinado}

BRASIL. Acre: Basin of Rio Purus, near of Mouth of Rio Macahuan (tributary of Rio Yaco), on terra firme, 21 Aug 1933, B.A. Krukoff 5590 (NY, US).

BOLIVIA. Santa Cruz: Ichilo, Parque Nacional Amboró, Río Saguayo, 21-I-1988, M. Saldías Paz 141 (NY, USZ).

PERÚ. Uyacali: Purús, rio Curanja, $10^{\circ} 04^{\prime} \mathrm{S}$, 7106'W, 250 m, 23 Oct 1997, J. Graham \& J. Schunke Vigo 255 (F, INPA).

\section{AGRADECIMIENTOS}

Este trabajo es parte de la revisión del género llevada a cabo por la primera autora, realizada mediante una beca doctoral de CONICET. Se agradece a los curadores de los herbarios consultados por permitir al segundo autor revisar las colecciones a su cuidado: BR, F, G, K, MG, MO, NY y USZ. Al Instituto de Botánica del Nordeste por facilitar el acceso a https://plants.jstor.org/. Se agradece al editor y revisores por las valiosas sugerencias.

\section{BIBLIOGRAFÍA}

Mouly, A.; S. G. Razafimandimbison, A. Khodabandeh \& B. Bremer. 2009. Phylogeny and classification of the speciesrich pantropical showy genus Ixora (Rubiaceae-Ixoreae) with implications of geographical monophyletic units and hybrids American Journal of Botany 96: 686-706.

Razafimandimbison, S. G. \& B. Bremer. 2002. Phylogeny and classification of Naucleeae s.l. (Rubiaceae) inferred from molecular (ITS, $r b c L$, and trnT-F) and morphological data. American Journal of Botany 89: 1027-1041.

Ridsdale, C. E. 1976. A revision of the tribe Cephalantheae (Rubiaceae). Blumea 23(1): 177-188.

Schumann, K. M. 1889. Tribus X. Naucleeae. In C. F. P. von Martius, A. G. Eichler, and I. Urban [eds.], Flora brasiliensis, vol. 6, 126-131. Fleischer, Leipzig, Germany.

Standley, P. C. 1931. The Rubiaceae of Bolivia. Publications of the Field Museum of Natural History. Botanical series 7(3): 255-339.

Standley, P. C. 1936. Rubiaceae, en: J.F. Macbride, Flora of Peru. Publications of the Field Museum of Natural History. Botanical series 13(6,1): 3-261.

Taylor, C. M.; J. A. Steyermark, P. G. Delprete, A. Vincentini, R. Cortés, D. C. Zappi, C. H. Persson, C. B. Costa \& E. Anunciação. 2004. Rubiaceae. 8: 497-847. In J. A. Steyermark, P. E. Berry \& B. K. Holst (eds.) Fl. Venez. Guayana. Missouri Botanical Garden Press, St. Louis.

Taylor, C. M. 2012. Ixora L. - In: Davidse G, Sousa Sánchez M, Knapp S, Chiang Cabrera F. (Eds), Flora Mesoamericana, Vol 4, págs. 263-266. Missouri Botanical Garden Press, St. Louis, Missouri,

Thiers, B. [permanentemente actualizado, consultado 2019]. Index Herbariorum: a global directory of public herbaria and associated staff. New York Botanical Garden's Virtual Herbarium, http://sweetgum.nybg.org/ih

Tropicos.org. Missouri Botanical Garden. [consultado Abril 2019] <http://www.tropicos.org > 